\title{
Bis-perfluorocycloalkenyl (PFCA) aryl ether monomers towards a versatile class of semi-fluorinated aryl ether polymers
}

\author{
Babloo Sharma ${ }^{a}$, Donald G. VanDerveer ${ }^{\mathrm{b}}$, Shawna M. Liff ${ }^{\mathrm{c}}$, Dennis W. Smith Jr. ${ }^{\mathrm{a}, *}$ \\ a Department of Chemistry and The Alan G. MacDiarmid NanoTech Institute, The University of Texas, 800 West Campbell Rd., BE 26, Ricahrdson, TX 75080, USA \\ ${ }^{\mathrm{b}}$ Department of Chemistry, Clemson University, Clemson, SC 29631, USA \\ ' Intel Corporation, Chandler, AZ 85226, USA
}

\section{A R T I C L E I N F O}

\section{Article history:}

Received 7 March 2013

Revised 17 April 2013

Accepted 19 April 2013

Available online 27 April 2013

\section{Keywords:}

Perfluoro and semifluorinated polymers

Perfluorocycloalkenes

Addition-elimination reaction

Polycondensation

\begin{abstract}
A B S T R A C T
A unique class of perfluorocycloalkenyl (PFCA) aryl ether monomers was synthesized from commercially available perfluorocycloalkenes (PFCAs) and bisphenols in good yields. This facile one pot reaction of perfluorocycloalkenes, namely, octafluorocyclopentene (OFCP), and decafluorocyclohexene (DFCH), with bisphenols occurs at room temperature via an addition-elimination reaction in the presence of a base. The synthesis of PFCA monomers and their condensation with bisphenols lead to perfluorocycloalkenyl (PFCA) aryl ether homopolymers and copolymers with random and/or alternating polymer architectures. Published by Elsevier Ltd.
\end{abstract}

Perfluorinated and semi-fluorinated polymers are continually sought due to their profound and exclusive properties in applied chemistry and biochemistry. ${ }^{1-4}$ Innovative and efficient methods are highly desired to strategically incorporate fluorine into molecular architectures with outstanding and specifically designed performance. Incorporation of fluorine into polymeric systems results in a unique combination of properties such as excellent thermal stability, chemical inertness, unique surface properties, low refractive index, low dielectric constant, and low optical loss in telecom wavelength. ${ }^{1,2,5-10}$ Such materials are potentially suitable as matrices for non-linear optics (NLO) or electro-optics (EO) applications as demonstrated by Iacono et al, and many other research groups including ours with perfluorocyclobutyl (PFCB) aryl ether polymers and other fluorinated vinylene derived polymers. ${ }^{8-11}$

Perfluorocycloalkenes (PFCA) are a general class of compounds comprising many commercially available perfluorocyclo-olefins including octafluorocyclopentene (OFCP) and decafluorocyclohexene (DFCH) (Fig. 1). As expected, PFCAs react with various nucleophiles including phenoxides, ${ }^{12-15}$ alkoxide, ${ }^{16-18}$ organometallic, ${ }^{19-}$ 21 amines, ${ }^{22-25}$ thiols, ${ }^{26}$ azoles $^{27}$ and phosphonium ylides. ${ }^{28}$ PFCA derivatives have thus shown a variety of applications including photochromic small molecules, ${ }^{29-33}$ nonlinear optical activity, ${ }^{34}$ lubricants, ${ }^{21}$ and etching agents. ${ }^{35}$ Herein we extend our recently published initial discovery of perfluorocyclopentenyl (PFCP) aryl ether polymers ${ }^{5}--$ a new step growth Polycondensation and report

\footnotetext{
* Corresponding author. Tel.: +001 972883 2915; fax: +001 9728832925 .

E-mail address: dwsmith@utdallas.edu (D.W. Smith).
}

the synthesis of bis-perfluorocycloalkenyl (PFCA) aryl ether monomers from perfluorocyclo-olefins, namely, OFCP and DFCH and bisphenols via an addition-elimination reaction in the presence of a base (Scheme 1 ).

Table 1 denotes the bisphenol and PFCA starting materials, monomers there from (M1-M6), and isolated yield of the condensation reaction. Further, the X-ray crystal structures of M1 (Fig. 2) and M6 (Fig. S22) were also solved.

With few exceptions such as, fluorinated vinylene derived Teflon, perfluorocyclco-olefins, in general, do not undergo homopolymerization via free radical mechanism. ${ }^{36}$ Recently, we have

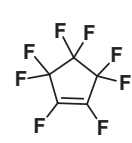

(a)

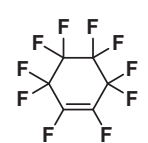

(b)
Figure 1. Commercially available perfluorocycloalkenes (a) OFCP and (b) DFCH.

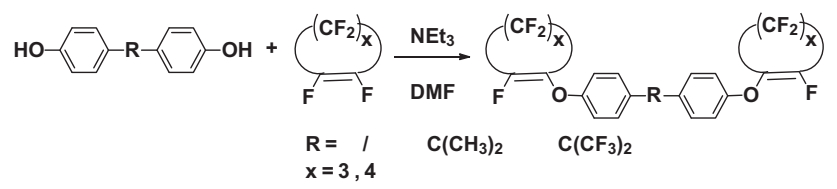

Scheme 1. Perfluorocycloalkenyl (PFCA) aryl ether monomer synthesis. 
Table 1

Reaction of bisphenols with perfluorocycloalkenes

\begin{tabular}{llll}
\hline Entry & Bisphenol & PFCA & Yield $^{\mathrm{a}}(\%)$ \\
\hline M1 & Biphenol & OFCP & 76 \\
M2 & Bisphenol-A & OFCP & 71 \\
M3 & Bisphenol-AF & OFCP & 72 \\
M4 & Biphenol & DFCH & 69 \\
M5 & Bisphenol-A & DFCH & 63 \\
M6 & Bisphenol-AF & DFCH & 62 \\
\hline
\end{tabular}

a Based on isolated product.

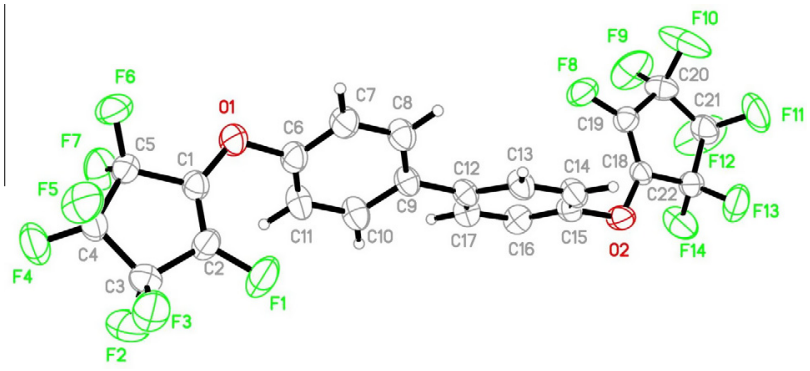

Figure 2. ORTEP representation of M1 crystal structure.

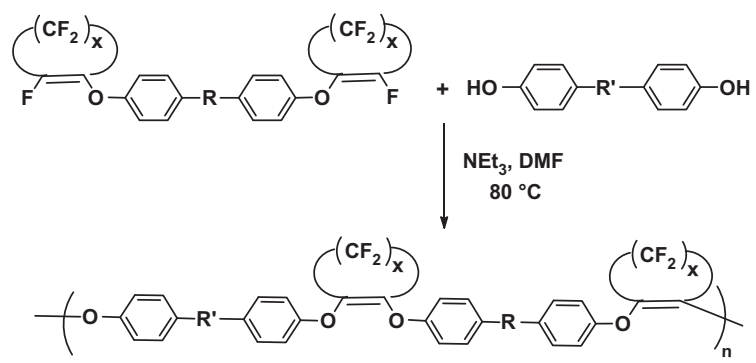

Scheme 2. General synthesis of PFCA aryl ether polymers from PFCA aryl ether monomers.

published the first polymerization of PFCAs using a novel step growth condensation with bisphenols. ${ }^{5}$ PFCA aryl ether polymers are highly thermally stable and show variable thermal properties depending upon the functional spacer group, $\mathrm{R}$ (Scheme 2).

Due to the volatility of perfluorocycloalkenes, the stochiometry ratio is difficult to sustain during polymerization. However, bisPFCA aryl ether monomers reported here, not only precludes the stochiometry ratio issue, but also provides a new synthetic versatility which can afford a variety of random and/or alternating copolymers with specific design control to be published elsewhere (Scheme 2). ${ }^{5}$ These monomers also provide the option of synthesizing copolymers containing both, OFCP and DFCP moieties.

Attack of the nucleophile on the PFCA double bond generates a carbanion which can eliminate fluoride resulting in vinyl substituted and allyl substituted products (Scheme 3 ). The ratio of vinylic to allylic products depends on the ring size, reaction conditions and nucleophile. ${ }^{26}$ The vinylic product would be the major product unless the allyl position has a more favored leaving group. ${ }^{26}$ The

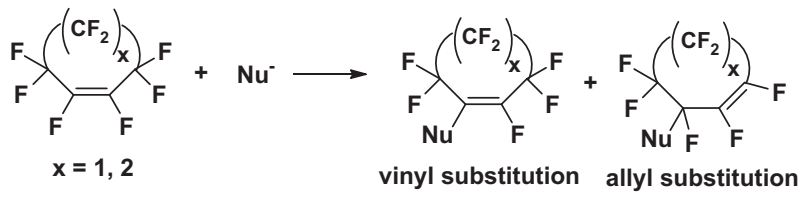

Scheme 3. General reaction representation for addition-elimination reactions on perfluorocycloalkenes. allylic product was noticeable in case of DFCH (M3-M6) as evident by three signals with identical molecular weight in the GC-MS spectra (Fig. S23). Allyl substitution was not observed in case of OFCP (M1-M3) as evidenced by NMR and GC-MS. The presence of allylic products for M4-M6 also explains the unaccounted signals in the ${ }^{1} \mathrm{H},{ }^{19} \mathrm{~F}$ and ${ }^{13} \mathrm{C}$ NMR (Figs. 3 and S13-S20). Interestingly, there were no other addition products, as has previously been reported with fluorinated arylene vinylene ether (FAVE) polymers. ${ }^{37,38}$

${ }^{13}$ Carbon ( ${ }^{19} \mathrm{~F}$-coupled) NMR has been proved to be a very helpful technique to determine structural properties of M1-M6. ${ }^{19}$ Fluorine coupled ${ }^{13}$ Carbon NMR of perfluorocycloalkenes and their products show short range as well as long range coupling, and each carbon of PFCA ring shows a splitting pattern due to each fluorine atom present on the ring. For example, in OFCP the $\beta$ carbon, a, with two fluorine atoms shows a tquint.t with $J=1375.0,120.0$, $25.4 \mathrm{~Hz}$, and the $\alpha$ carbon, b, shows a tqm, $J=1287.5,116.8 \mathrm{~Hz}$ (Fig. S2). As expected, DFCH and its products show a more complicated and unresolved splitting pattern in their ${ }^{13} \mathrm{C}$ NMR spectra due to the higher number of fluorine atoms (Figs. S4, S15, S18 and S20).

In the case of M1, the ${ }^{1} \mathrm{H}$ NMR spectrum (Fig. S5) shows a doublet of doublet (at 7.41 and 7.67 with $J=8.2 \mathrm{~Hz}$ ), representing a symmetric environment. The ${ }^{19} \mathrm{~F}$ NMR (Fig. S6) shows well integrated four fluorine peaks, where peak at $-150.45 \mathrm{ppm}$ represents the terminal vinyl fluorine indicating the addition-elimination reaction product. The ${ }^{13} \mathrm{C}$ NMR $\left({ }^{19} \mathrm{~F}\right.$ coupled) spectrum (Fig. 4) shows singlets for the phenylene carbons whereas PFCP ring carbon signals undergo short range as well as long range coupling and therefore a complex pattern is observed. For example, the allylic carbon, f, shows ttd with $J=1032.1,98.5,27.7 \mathrm{~Hz}$ whereas the $\beta$ carbon, e, shows a broad splitting pattern overlapping with adjacent carbon, g's, signals.

To further establish the structures and understand the solidstate nature of these monomers, X-ray analysis was conducted. Bis-PFCA monomers were purified using column chromatography with hexanes as the eluent. The purified monomers, M1, M4 and M6 were obtained as white crystalline solids, while other monomers were highly viscous clear liquids. Attempts to crystallize M1, M4 and M6 from a multitude of polar and non-polar solvents were unsuccessful. Finally, recrystallization of M1 and M6 from chlorobenzene and a mixture of toluene and bromobenzene, respectively, with a slow evaporation, resulted in the afforded Xray quality crystalline solids. Crystal structures for M1 and M6 are shown in Figures 2 and S22, and selected crystal properties including dihedral bond angles $\left(^{\circ}\right)$ for $\mathbf{M 1}$ and $\mathbf{M 6}$ are reported in Tables 2 and S1, respectively.

Figure 2 shows the ORTEP representation for M1. Crystal structure measurements were carried out at $193 \mathrm{~K}$. The crystal shows the monoclinic, P21/n space group with no sign of thermal atomic displacement. Both PFCP rings show a presence of a double bond (C1-C2 and C18-C19) with a bond length of $1.32 \AA$. Two phenyl rings stand with a torsion angle (C8-C9-C12-C13) of $30.8^{\circ}$. The PFCP ring (with higher numbered carbon) inclined to the phenyl ring with $90.3^{\circ}$, but the other PFCP ring inclined with $56.6^{\circ}$ to the corresponding phenyl ring. Interestingly, the former PFCP ring is almost planar, but PFCP ring (with lowered numbered carbon) shows an envelope conformation with the flap atom, C4.

Figure S22 shows the ORTEP representations for molecule A and molecule B, two conformation structures of M6 crystal. Crystal data were recorded at $188 \mathrm{~K}$. The crystal shows triclinic, P-1 space group and a significant amount of thermal displacements, resulting in the asymmetry in molecules. $\mathbf{M 6}$ crystal properties are very different from M1 crystal structural properties because of two extra trifluoromethyl groups and a bigger PFCA rings. Both molecules A and $\mathrm{B}$ show the $\mathrm{C} \equiv \mathrm{C}$ bond length of $1.32 \AA$ in $\mathrm{PFCH}$ rings as we 


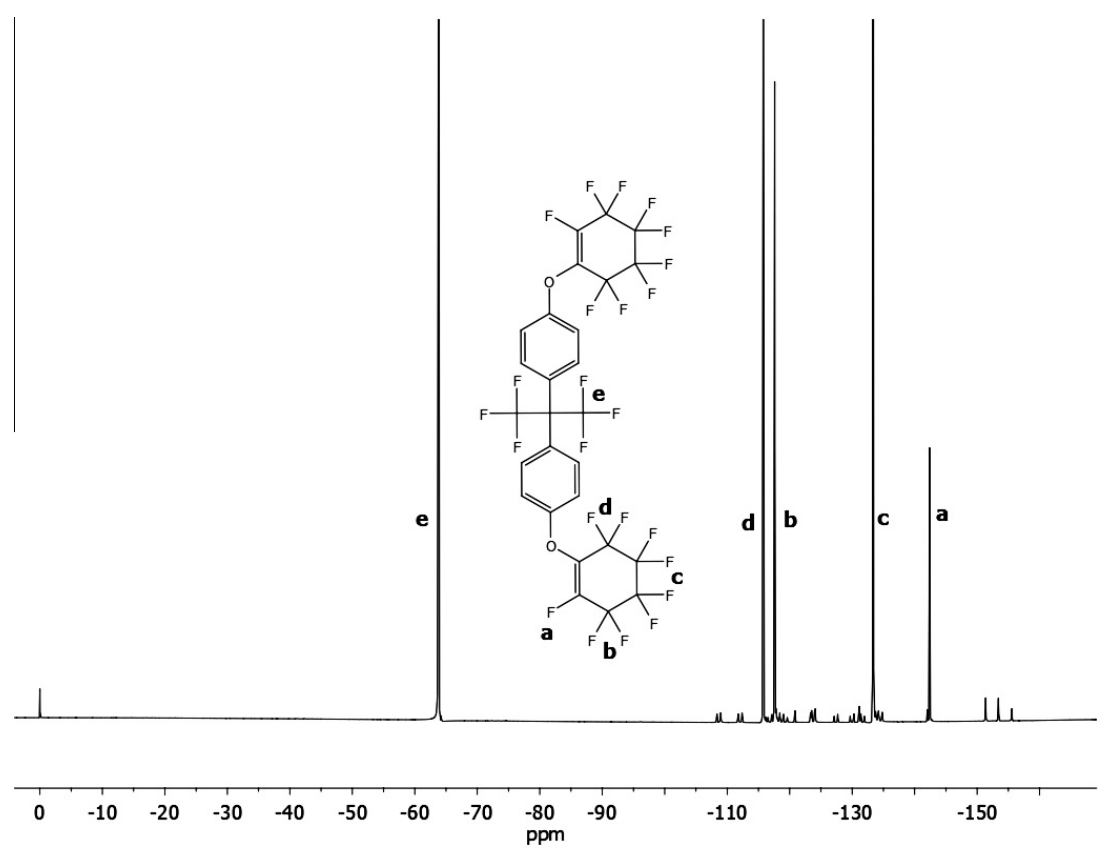

Figure 3. ${ }^{19} \mathrm{~F}$ NMR of monomer M6. Minor peaks represent the allyl substituted products.

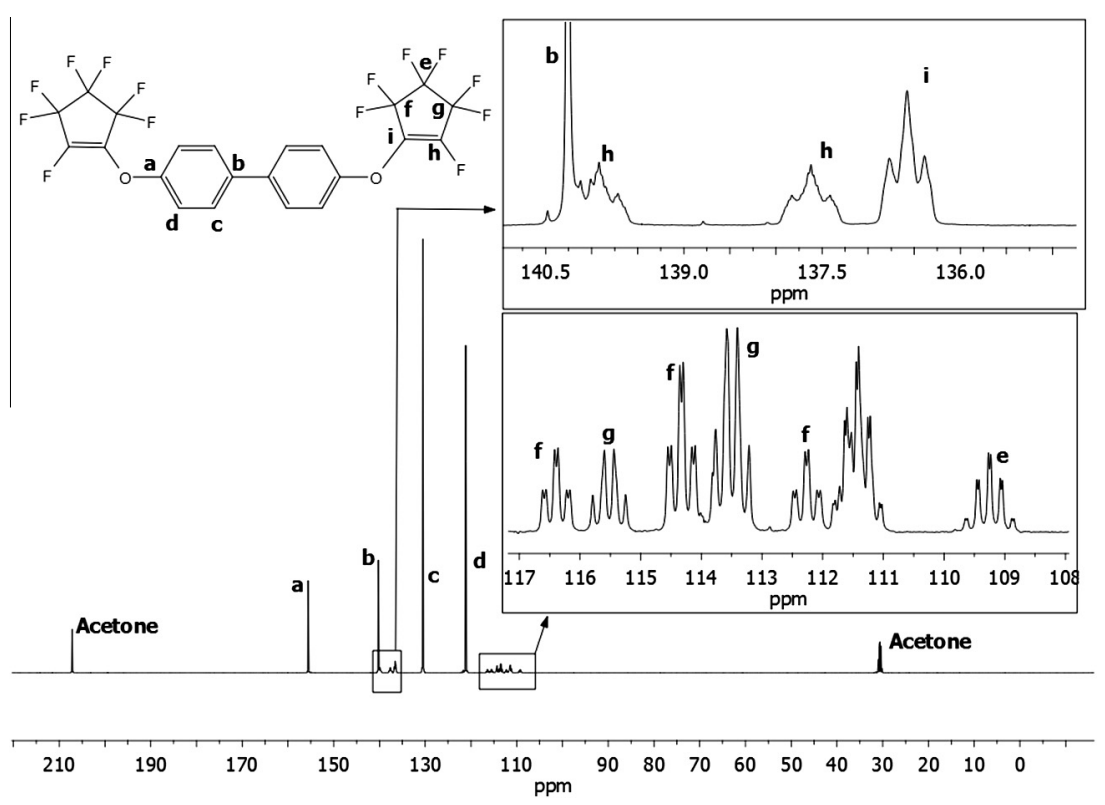

Figure 4. ${ }^{13} \mathrm{C}$ NMR $\left({ }^{19} \mathrm{~F}\right.$-coupled) of monomer M1. Insets: expanded signals of PFCP ring carbons.

Table 2

Selected crystal data and structure refinements for monomer M1

\begin{tabular}{lc}
\hline Parameters & M1 $\left(^{\circ}\right)$ \\
\hline C8-C9-C12-C13 & 30.8 \\
C1-O1-C6-C11 & 56.6 \\
C18-O2-C15-C14 & 90.3 \\
C2-C1-C5-C4 & 10.2 \\
C18-C19-C20-C21 & -1.5 \\
\hline
\end{tabular}

see in M1 crystal. The PFCH rings inclined to the corresponding phenyl rings with an angle ranging from $62^{\circ}$ to $74^{\circ}$. In molecule A the high numbered PFCH rings shows half chair geometry with no sign of atomic displacement whereas the low numbered PFCH ring shows distorted chair to distorted half chair conformation with atom C14 distorted over two sites (C14 and C14A), and concomitant disorder of $\mathrm{F}$ atoms on the ring. In molecule $\mathrm{B}$, both $\mathrm{PFCH}$ rings show the half chair conformation; with the high numbered PFCH ring showing the atomic thermal displacement (F23' and F23B).

These PFCA monomers (M1-M6) were also characterized with ATR-FTIR. Figures 5 and S21 show the IR spectrum for monomers M1-M6. A strong peak at $830 \mathrm{~cm}^{-1}$ represents the typical, out of plane, aromatic $\mathrm{C}-\mathrm{H}$ bending mode. ${ }^{39}$ These spectra show the characteristic bands from 1080 to $1285 \mathrm{~cm}^{-1}$ due to $\mathrm{CF}_{2}$ and $\mathrm{C}-\mathrm{O}-\mathrm{C}$ asymmetric stretching. ${ }^{35,40}$ Peaks at 1499 and $1600 \mathrm{~cm}^{-1}$ (strong and medium intensities respectively) are associated with $\mathrm{C}=\mathrm{C}$ stretching for the aromatic rings in these spectra. Figures 5 and 


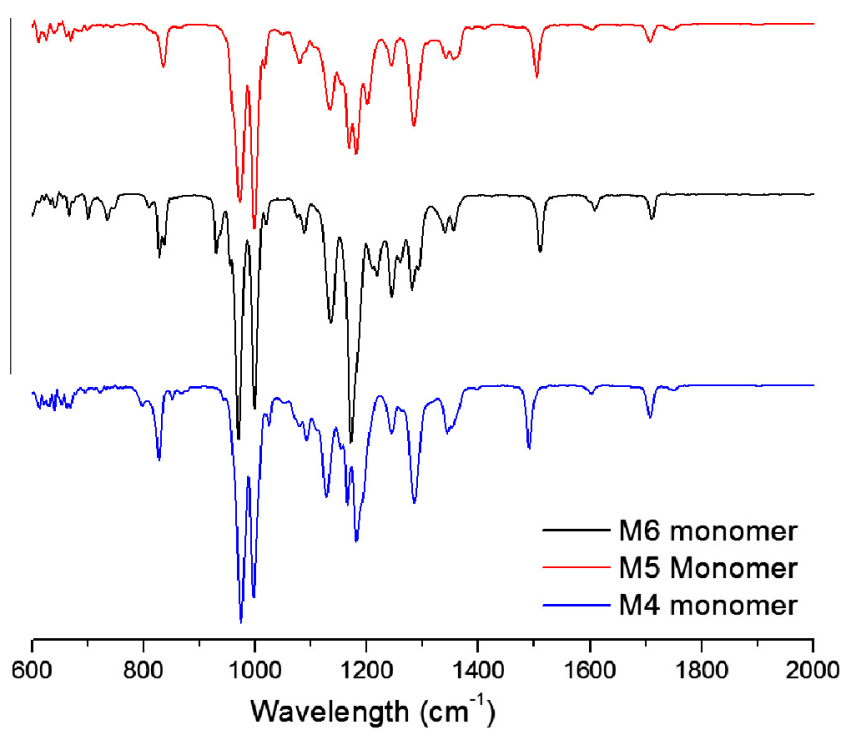

Figure 5. FTIR-IR spectra for M4-M6.

S21 show $\mathrm{C}=\mathrm{C}$ stretching peaks of the $\mathrm{PFCP}$ and $\mathrm{PFCH}$ rings at 1724 and $1707 \mathrm{~cm}^{-1}$, respectively. ${ }^{18,41}$ Also Figure 5 shows a small peak at $1747 \mathrm{~cm}^{-1}$ (easily visible for M4 and $\mathbf{M 6}$ ) which belongs to characteristic $\mathrm{C}=\mathrm{C}$ stretching for a allylic substituted $\mathrm{PFCH}$ ring (minor products). ${ }^{18}$ Further, the GC-MS and elemental analyses showed the expected molecular weight and elemental composition for all the products M1-M6.

In conclusion, we have developed a new class of PFCA aryl ether monomers from commercially available feedstocks via an addition-elimination reaction, in a good isolated yield. These monomers are a substantial step for the synthesis of a wide range of PFCA homo and copolymers of variable randomness or blockiness.

\section{Acknowledgments}

The authors want to express their gratitude to Intel Corporation (Grant \#4606175), Welch Foundation (Grant \#AT-0041), NSFIUCRC sponsored Center for Energy Harvesting Materials and Systems (CEHMS) (Grant \#IIP-1035024) and the University of Texas at Dallas for their financial support.

\section{Supplementary data}

Supplementary data (crystallographic data for the structures reported in this Letter have been deposited with the Cambridge Crystallographic Data Centre as supplementary publications CCDC 927350 (1) and 927351 (2). Copies of the data can be obtained free of charge on application to CCDC, 12 Union Road, Cambridge CB2 1EZ, UK (fax: +44 1233336 033: e-mail: deposit@ccdc.cam.ac.uk). A list of detailed experimental procedures, instrumentation and characterization data (NMR, IR, GC/MS) for all the new compounds) associated with this article can be found, in the online version, at http://dx.doi.org/10.1016/j.tetlet.2013.04.087.

\section{References and notes}

1. Ameduri, B., Boutevin, B., Eds.Well-Architectured Fluoropolymers: Synthesis, Properties and Applications; Elsevier Science: San Diego, 2004.

2. Scheirs, J., Ed.Modern Fluoropolymers: High Performance Polymers for Diverse Applications; Wiley: Chichester, 1997.

3. Feiring, A. E., Ed.Organofluorine Chemistry: Principles and Commercial Applications; Banks, R. E., Smart, B. E., Tatlow, J. C., Eds.; Plenum Press: New York, 1994.

4. Biomedicinal Aspects of Fluorine Chemistry; Filler, R., Kobayashi, Y., Eds.; Elsevier: Amsterdam, 1982.

5. Cracowski, J.-M.; Sharma, B.; Brown, D. K.; Cristensen, K.; Lund, B. R.; Smith, D. W., Jr. Macromolecules 2012, 45, 766.

6. Hougham, G. G.; Cassidy, P. E.; Johns, K.; Davidson, T. Fluoropolymers: Synthesis; Plenum Pub Corp: New York, 1999.

7. Keck, S.; Knoerzer, T. A.; Smith, D. W., Jr.; Iacono, S. T. Polym. Int. 2013. http:// dx.doi.org/10.1002/pi.4447.

8. Kettwich, S. C., Lund, B. R., Smith, D. W., Jr., Iacono, S. T., Eds.Advances in Fluorine-Containing Polymers; American Chemical Society: Washington, DC, 2012; Vol. 1106, p 9

9. Iacono, S. T.; Budy, S. M.; Moody, J. D.; Smith, R. C.; Smith, D. W., Jr. Macromolecules 2008, 41, 7490 .

10. For a review see: Iacono, S. T.; Budy, S. M.; Jin, J.; Smith, D. W., Jr. J. Polym. Sci., Part A: Polym. Chem. 2007, 45, 5705.

11. Ma, H.; Jen, A. K. Y.; Dalton, L. R. Adv. Mater. 2002, 14, 1339.

12. Lindner, P. E.; Lemal, D. M. J. Org. Chem. 1996, 61, 5109.

13. Bayliff, A. E.; Bryce, M. R.; Chambers, R. D. J. Chem. Soc., Perkin Trans. 1 1987, 763.

14. Stockel, R. F.; Beachem, M. T.; Megson, F. H. J. Org. Chem. 1965, 30, 1629.

15. Stockel, R. F.; Beachem, M. T.; Megson, F. H. Can. J. Chem. 1964, 42, 2880.

16. Plevey, R. G.; Talbot, R. E. J. Fluorine Chem. 1977, 10, 577-584.

17. Plevey, R. G.; Sparrow, D. J. J. Chem. Soc., Perkin Trans. 1 1976, 573-577.

18. Clayton, A. B.; Roylance, J.; Sayers, D. R.; Stephens, R.; Tatlow, J. C. J. Chem. Soc. 1965,7358

19. Yamada, S.; Konno, T.; Ishihara, T.; Yamanaka, H. J. Fluorine Chem. 2005, 126, 125-133.

20. Han, M.; Lee, S.; Jung, J.; Park, K.; Kwon, S.; Ko, J.; Lee, P. H.; Kang, Y. Tetrahedron 2006, 62, 9769-9777.

21. Organometallic derivatives of perfluorocycloalkenes. U.S. Patent $3,787,461$, 1974.

22. Powers, G. A.; Stephens, R.; Tatlow, J. C. J. Fluorine Chem. 1982, 20, 555-558.

23. McBee, E. T.; Turner, J. J.; Morton, C. J.; Stefani, A. P. J. Org. Chem. 1965, 30 3698-3705.

24. Cullen, W. R.; Dhaliwal, P. S. Can. J. Chem. 1967, 45, 719.

25. Mir, Q. C.; Guo, C. Y.; Kirchmeier, R. L.; Shreeve, J. M. J. Org. Chem. 1994, 59, 173

26. Timperley, C. M. J. Fluorine Chem. 2004, 125, 685-693.

27. Garg, S.; Twamley, B.; Zhuo, Z.; Shreeve, J. M. Chem. Eur. J. 2009, 15, 10554.

28. Yamada, S.; Ishii, E.; Konno, T.; Ishihara, T. Org. Biomol. Chem. 2007, 5, 1442.

29. Irie, M.; Sakemura, K.; Okinaka, M.; Uchida, K. J. Org. Chem. 1995, 60, 8305.

30. Wigglesworth, T. J.; Sud, D.; Norsten, T. B.; Lekhi, V. S.; Branda, N. R. J. Am. Chem. Soc. 2005, 127, 7272.

31. Malval, J.-P.; Gosse, I.; Morand, J.-P.; Lapouyade, R. J. Am. Chem. Soc. 2002, 124 904.

32. Yamada, T.; Muto, K.; Kobatake, S.; Irie, M. J. Org. Chem. 2001, 66, 6164

33. Yamada, T.; Kobatake, A.; Muto, K.; Irie, M. J. Am. Chem. Soc. 2000, 122, 1589.

34. Matsui, M.; Tsuge, M.; Funabiki, K.; Shibata, K.; Muramatsu, H.; Hirota, K.; Hosoda, M.; Tai, K.; Shiozaki, H.; Kim, M.; Nakatsu, K. J. Fluorine Chem. 1999, 97, 207-212.

35. Takahashi, K.; Itoh, A.; Nakamura, T.; Tachibana, K. Thin Solid Films 2000, 374 303.

36. Beale, J. H.; Schwartz, N. N.; Mantell, G. J. J. Polym. Sci., Polym. Lett. Ed. 1978, 16, 67.

37. Moody, J. D.; VanDerveer, D.; Smith, D. W., Jr.; Iacono, S. T. Org. Biomol. Chem. 2011, 9, 4842 .

38. Iacono, S. T.; Budy, S. M.; Ewald, D.; Smith, D. W., Jr. Chem. Commun. 2006 4844.

39. Silverstein, R. M.; Webster, F. X. Spectroscopic Identification of Organic Compounds; Wiley: India, 2006

40. Cotthup, N. B.; Daly, L. H.; Wiberley, S. E. Introduction to Infrared and Raman Spectroscopy; Academic Press: New York, 1964.

41. Vasekova, E.; Drage, E. A.; Smith, K. M.; Mason, N. J. J. Quant. Spectrosc. Radiat. Transfer 2006, 102, 418. 